\title{
Clinical Study \\ Results of a Second Year of Therapy with the 12-Month Histrelin Implant for the Treatment of Central Precocious Puberty
}

\author{
Samar Rahhal, ${ }^{1}$ William L. Clarke, ${ }^{2}$ Gad B. Kletter, ${ }^{3}$ Peter A. Lee,, 4 \\ E. Kirk Neely, ${ }^{5}$ Edward O. Reiter, ${ }^{6}$ Paul Saenger, ${ }^{7}$ Dorothy Shulman, ${ }^{8}$ \\ Lawrence Silverman, ${ }^{9}$ and Erica A. Eugster ${ }^{1}$ \\ ${ }^{1}$ Department of Pediatrics, Riley Hospital for Children, Indiana University School of Medicine, Indianapolis, IN 46202, USA \\ ${ }^{2}$ Department of Pediatrics, University of Virginia Health Sciences Center, Charlottesville, VA 22908, USA \\ ${ }^{3}$ Department of Pediatrics, Seattle Childrens Hospital and Medical Center, Seattle, WA 98105-0371, USA \\ ${ }^{4}$ Department of Pediatrics, Pennsylvania State Children's Hospital, Hershey, PA 17033, USA \\ ${ }^{5}$ Department of Pediatrics, Stanford University Medical Center, Stanford, CA 94305, USA \\ ${ }^{6}$ Department of Pediatrics, Baystate Children's Hospital, Springfield, MA 01199, USA \\ ${ }^{7}$ Department of Pediatrics, Montefiore Medical Center, Albert Einstein College of Medicine, Bronx, NY 10467, USA \\ ${ }^{8}$ Department of Pediatrics, University of South Florida and All Children's Hospital, St. Petersburg, FL 33701, USA \\ ${ }^{9}$ Department of Pediatrics, Goryeb Children's Hospital Atlantic Health, Morristown, NJ 07962, USA
}

Correspondence should be addressed to Erica A. Eugster, eeugster@iupui.edu

Received 10 November 2008; Revised 29 January 2009; Accepted 11 February 2009

Recommended by Juliane Leger

\begin{abstract}
Background. Gonadotropin releasing hormone analogs (GnRHas) are standard of care for central precocious puberty (CPP). The histrelin subcutaneous implant is safe and effective in the treatment of CPP for one year. Objective. The study evaluates a second year of therapy in children with CPP who received a new implant after one year of treatment. Methods. A prospective one-year study following an initial 12-month treatment period was conducted. Results. Thirty-one patients (29 girls) aged $7.7 \pm 1.5$ years received a second implant. Eighteen were naïve to GnRHa therapy at first implantation. Peak LH declined from $0.92 \pm 0.58 \mathrm{mIU} / \mathrm{mL}$ at 12 months to $0.51 \pm 0.33 \mathrm{mIU} / \mathrm{mL}$ at 24 months $(P<.0001)$ in naïve subjects, and from $0.74 \pm 0.50 \mathrm{mIU} / \mathrm{mL}$ at $12 \mathrm{months}$ to $0.45 \pm 0.35 \mathrm{mIU} / \mathrm{mL}$ at 24 months $(P=.0081)$ in previously treated subjects. Predicted adult height increased by $5.1 \mathrm{~cm}$ at 24 months $(P=.0001)$. Minor implant site reactions occurred in $61 \%$, while minor difficulties with explantation occurred in $32.2 \%$ of subjects. Conclusion. The histrelin implant demonstrates profound hypothalamic-pituitary-gonadal axis suppression when a new implant is placed for a second year of treatment. Prospective follow-up of this therapeutic modality for the treatment of CPP is needed.
\end{abstract}

Copyright (C) 2009 Samar Rahhal et al. This is an open access article distributed under the Creative Commons Attribution License, which permits unrestricted use, distribution, and reproduction in any medium, provided the original work is properly cited.

\section{Introduction}

Central precocious puberty $(\mathrm{CPP})$ is defined as activation of the hypothalamic-pituitary-gonadal (HPG) axis before the age of 8 years in Caucasian girls and 7.5 years in African American girls, and before the age of 9 years in boys [1]. Treatment is often initiated with the goal of preserving final adult height. Gonadotropin releasing hormone analogs (GnRHa) have been the cornerstone of treatment of CPP for more than 20 years [2]. A depot form of GnRHa is traditionally used in the US and is administered as a monthly injection. Although known to be safe and effective [3-5], the need for intramuscular injections may be bothersome to patients and adversely affect compliance.

A one-year subcutaneous histrelin implant has emerged as an alternative approach for the treatment of CPP. Histrelin is a GnRHa which is 150 times more potent than endogenous GnRH. The implant is a flexible, non-biodegradable device that contains histrelin acetate and is inserted subcutaneously using a trocar. The microporous walls allow histrelin to diffuse at a rate of approximately $65 \mathrm{mcg} /$ day. A pilot study of 11 girls with CPP showed that the implant effectively suppressed the HPG axis [6]. This was followed by a phase III prospective trial involving 36 children which demonstrated 
TABLE 1: Patient characteristics at month 12 when the second implant was placed.

\begin{tabular}{|c|c|c|c|}
\hline & GnRHa naïve $(N=18)$ & GnRHA pretreated $(N=13)$ & All patients $(N=31)$ \\
\hline Age (years) & $8.0 \pm 1.4$ years & $9.6 \pm 1.3$ years & $8.7 \pm 1.6$ years $($ range, $5-11)$ \\
\hline \multirow{2}{*}{ Sex } & 18 Girls (100.0\%) & 11 Girls $(84.6 \%)$ & 29 Girls (93.5\%) \\
\hline & 0 Boys & 2 Boys $(15.4 \%)$ & 2 Boys $(6.5 \%)$ \\
\hline Weight (Kg) & $39.4 \pm 9.7$ & 51.3. \pm 12.9 & $44.4 \pm 12.5($ range, $24-72)$ \\
\hline Height SDS $(\mathrm{cm})$ & $1.7 \pm 1.14$ & $1.2 \pm 1.02$ & $1.5 \pm 1.10$ \\
\hline BMI (\%) & $88.3 \pm 17.6$ & $93.6 \pm 10.1$ & $90.6 \pm 14.9$ \\
\hline
\end{tabular}

BMI = body mass index.

safety and efficacy of the implant for one year [7]. Whether a second year of therapy with a new implant would have similar results is unknown. Thus, the objective of this study was to determine safety and efficacy of a second year of therapy with a new implant for the treatment of CPP.

\section{Methods}

2.1. Subjects. Patients from nine US medical centers who completed the one-year phase III trial were offered the option to have a second implant placed. Institutional review board approval was obtained at each site. Informed consent was obtained from parents or legal guardians, and assent from all children greater than 7 years of age. Inclusion criteria included patients in whom the decision was made to continue with GnRHa therapy.

2.2. Study Design. This was a one-year multicenter prospective open label trial. At all sites, a pediatric surgeon removed the first implant and inserted the second during the same procedure, using a standardized trocar device. The type of anesthesia used varied according to site, and included local anesthesia, conscious sedation, or general anesthesia. All implants contained $50 \mathrm{mg}$ of histrelin. Clinic visits occurred every 6 months. Height and weight were measured at every visit and annualized growth velocity was determined. GnRHa stimulation tests were performed at 1,6 , and 12 months after placement of the second implant. They were performed using a dose of $20 \mathrm{mcg} / \mathrm{kg}$ of aqueous leuprolide acetate and measuring $\mathrm{LH}$ and FSH at baseline, 30, and 60 minutes after GnRHa. Gonadotropin levels were measured by immunochemiluminometric assay (Esoterix, Calabasas Hills, Calif, USA). Stimulated levels of $\mathrm{LH}<4 \mathrm{mIU} / \mathrm{mL}$ and $\mathrm{FSH}<2.5 \mathrm{mIU} / \mathrm{mL}$ were considered suppressed (prepubertal references values; LH 0.02-3, FSH 1.0-4.2 in girls, 0.263.0 in boys). Estradiol and testosterone were measured by radioimmunoassay (Esoterix) at baseline only. Levels below $20 \mathrm{pg} / \mathrm{mL}(73.42 \mathrm{pmol} / \mathrm{L})$ for estradiol and $30 \mathrm{ng} / \mathrm{dl}$ $(0.69 \mathrm{nmol} / \mathrm{L})$ for testosterone were considered suppressed (prepubertal reference ranges; estradiol $<15 \mathrm{pg} / \mathrm{mL}$, testosterone $3-10 \mathrm{ng} / \mathrm{dL}$ ). Histrelin levels were measured at 1 , 6, and 12 months after placement of the second implant. Bone age radiographs were obtained annually and were centrally read (Lifespan Health Research Center, Kettering, Ohio, USA). Predicted adult heights were determined using

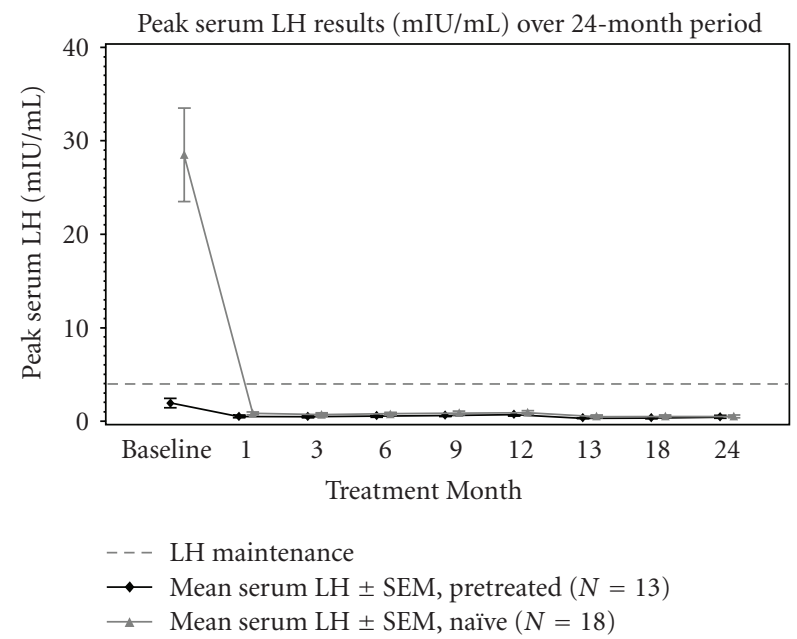

Figure 1: Peak LH in naïve and previously treated subjects from baseline through Month 24 .

the Bayley-Pinneau method [8], using the average rather than accelerated tables.

2.3. Statistics. Summary statistics were performed to derive baseline characteristics. Data are expressed as mean \pm standard deviation. All statistical tests were conducted using the student's paired $t$-test. All analysis and computations were performed using SAS version 9.1.3 (SAS Institute, Cary, NC, USA) on the Microsoft Windows XP platform (Richmond, Calif, USA).

\section{Results}

3.1. Subject Characteristics. Thirty one patients ( 29 girls) had a second implant placed. Of these, 18 had been naïve to GnRHa treatment prior to placement of the first implant and 12 had been previously treated. Patient characteristics are summarized in Table 1.

3.2. Hormonal Assessments. Peak LH in response to GnRHa stimulation remained suppressed in all subjects throughout the study period (Figure 1). It decreased from $0.92 \pm$ $0.58 \mathrm{mIU} / \mathrm{mL}$ at $12 \mathrm{months}$ to $0.51 \pm 0.33 \mathrm{mIU} / \mathrm{mL}$ at 24 months $(P<.0001)$ in the naïve group, and from $0.74 \pm$ $0.50 \mathrm{mIU} / \mathrm{mL}$ at 12 months to $0.45 \pm 0.35 \mathrm{mIU} / \mathrm{mL}$ at 


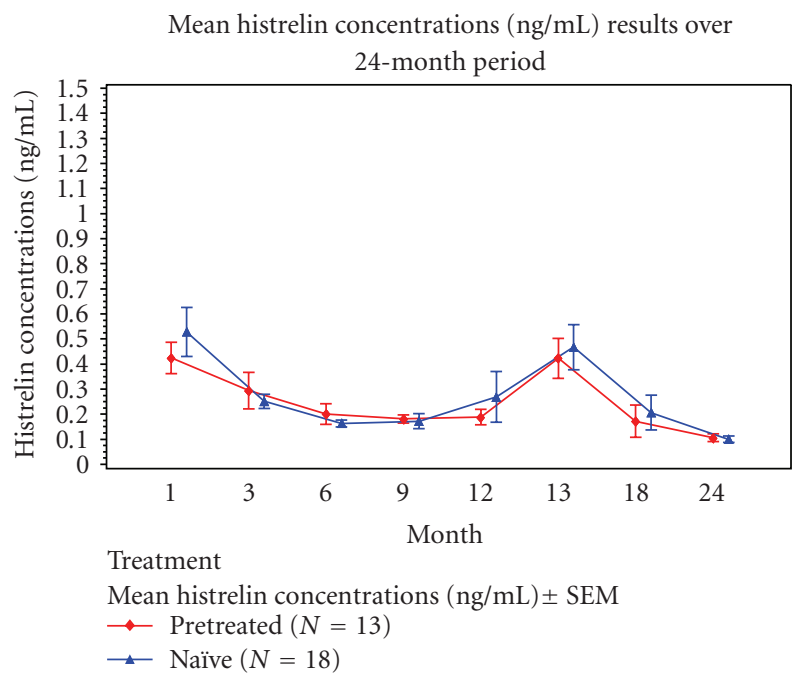

FIGURE 2: Histrelin levels throughout the 2 years of therapy.

24 months $(P=.0081)$ in previously treated subjects. Peak FSH in response to GnRHa stimulation at 24 months was not different than at 12 months and remained suppressed at $2.45 \pm 1.12 \mathrm{mIU} / \mathrm{mL}$ (naïve) and $2.40 \pm 1.11 \mathrm{mIU} / \mathrm{mL}$ (previously treated). Estradiol levels remained suppressed throughout the second year of therapy. Testosterone levels in the two boys at 24 months were $6.8 \mathrm{ng} / \mathrm{dL}$ and $13 \mathrm{ng} / \mathrm{dL}$, respectively. Histrelin levels were detectable throughout the second year of therapy and are shown in Figure 2.

3.3. Radiographic Studies and Growth Parameters. The average bone age to chronological age ratio decreased significantly in all patients from 1.29 at the time of the second implant to 1.23 at 24 months $(P<.0001)$ (Figure 3$)$. No difference in growth velocity SDS was seen at 24 months compared to 12 months in the naïve patients. In contrast, the growth velocity SDS decreased from $-1.50 \pm 2.70$ at 12 months to $-2.52 \pm 2.68$ at 24 months in the previously treated patients $(P=.005)$. One patient in the naïve group was started on growth hormone because of significant slowing of growth. As shown in Figure 4, average predicted adult height increased in both groups of girls at 24 months relative to baseline, resulting in a $5.1 \mathrm{~cm}$ increase in the group as a whole after two years of treatment, $(P<.0001)$. Mean predicted adult height for the two boys was $165.6 \mathrm{~cm}$ at baseline and $163.7 \mathrm{~cm}$ at 24 months $(P=.84)$. BMI percentiles decreased in all patients from $89.9 \pm 12.5 \%$ at baseline to $88.0 \pm 19.6 \%$ at 24 months $(P<.001)$. Tanner staging did not significantly change over the course of the year. Testicular volume was 3$6 \mathrm{~mL}$ in one boy and $6-10 \mathrm{~mL}$ in the other at 24 months, both of which were not different from 12 months.

3.4. Safety. Nineteen patients $(61 \%)$ reported minor implant site reactions, (e.g., bruising or pain) immediately after implant replacement. These were self-resolving and of brief duration. One patient developed a keloid. There were no cases of spontaneous implant extrusion or infection. There were some issues related to implant removal. At

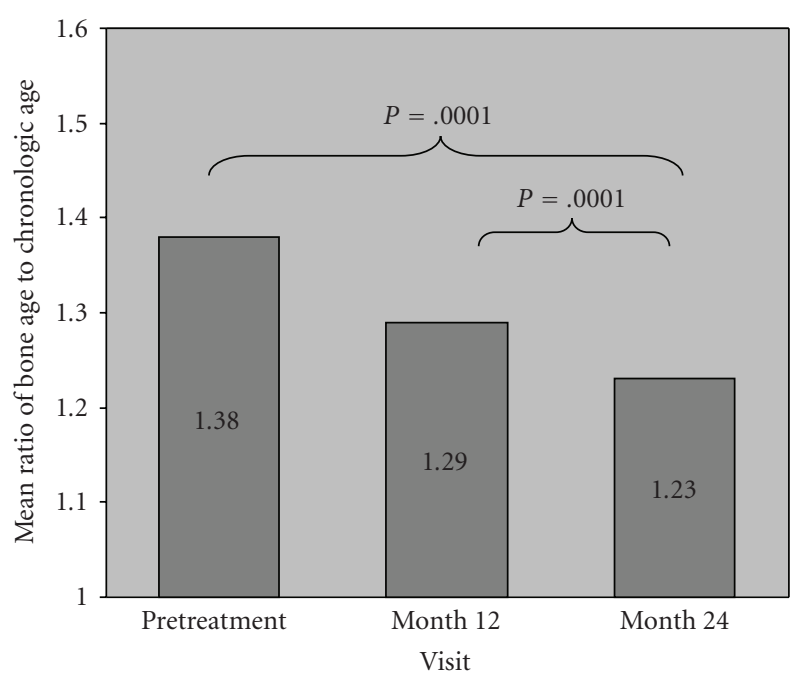

FIGURE 3: Ratio of bone age to chronological age at baseline, 12 and 24 months in all subjects.

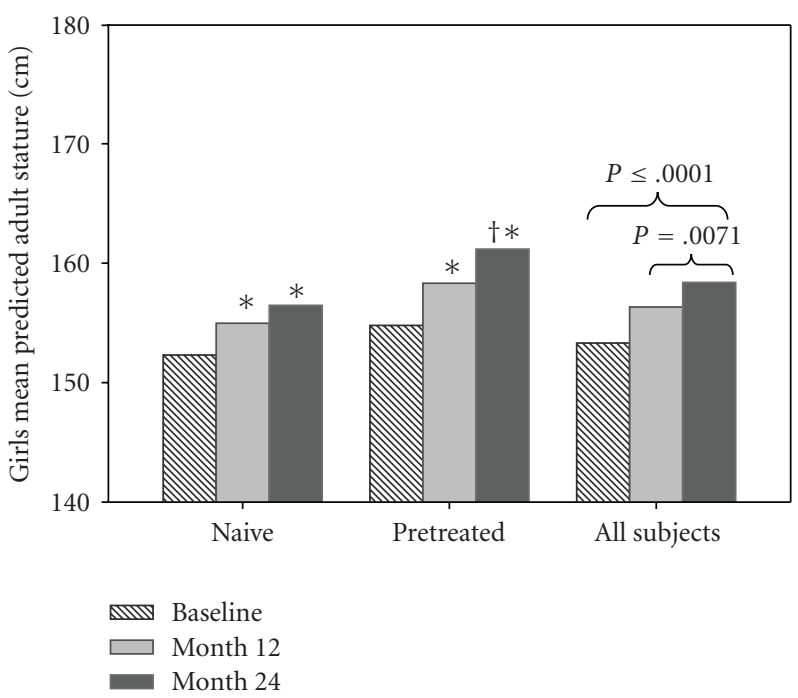

Figure 4: Predicted adult height at baseline, 12 and 24 months in both groups of subjects. ${ }^{*} P<.02$ compared to baseline, ${ }^{\dagger} P<.01$ compared to 12 months.

12 months, 8 patients (22.2\%) had implant breakage on removal and $2(5.6 \%)$ required ultrasound localization prior to explantation. At 24 months, 5 patients $(16.1 \%)$ had implant breakage on removal and 3 cases (9.6\%) required ultrasound localization.

\section{Discussion}

The treatment for CPP in the US has traditionally involved intramuscular monthly injections of leuprolide acetate. While a three-month depot leuprolide is available, it has not been adequately studied in children and failed to demonstrate equivalent short-term HPG axis suppression when compared to monthly injections [9]. Although experience is limited, the histrelin acetate implant appears to be an exciting 
novel therapy for CPP that obviates the need for injections. Following placement of a new implant at 12 months, HPG axis suppression was maintained in all patients throughout a second year of therapy. In addition, the rate of skeletal maturation slowed significantly and predicted adult heights improved after 24 months compared to baseline.

In terms of safety, the implant was well tolerated overall. Implant site reactions were mild, and minor difficulties with implant removal were reported either at 12 or 24 months in $\sim 20 \%$ of patients. These involved implant breakage and the need for ultrasound to localize the implant. While the implants were not found to be broken in situ, some broke on removal and this made the explant process more difficult and in some cases more time consuming. Implant breakage may be related to surgical technique and with improved experience, complications may be avoidable. Although no adverse events related to anesthesia have been reported in patients treated with the implant, the inherent risks of conscious sedation and general anesthesia in particular should be taken into consideration when these methods are used.

The main theoretical concern with the histrelin implant is the potential for adverse effects on growth, body composition, and the reproductive system from the even more profound HPG axis suppression than that seen with traditional therapy. Results from this study suggest that a decline in stimulated $\mathrm{LH}$ might occur in each successive year of treatment with this device. While this may raise concern regarding growth while on the implant, a recent study actually suggests that increased suppression of the HPG axis in children with CPP may result in improved growth velocity [10]. In our study, the growth velocity SDS did not change significantly in year 2 in the naïve group, but it did decline in the previously treated patients. However, this was likely related to the relatively advanced bone ages in this group of children at the time that the second implant was placed. Despite this, an improvement in predicted adult heights was seen in both groups of patients. Also reassuring was the small yet significant decrease in BMI noted at 24 months compared with 12 months. Finally, 4 of the 5 patients who did not have a second implant placed at 12 months returned for stimulated LH measurements at 13 months. HPG axis recovery was noted in all 4 subjects by that time. In addition, histrelin levels were undetectable by 13 months. Information from patients on therapy for longer than one year will be needed to determine whether timing of HPG axis recovery will be different in children treated with the implant as compared with traditional monthly injections.

In conclusion, the histrelin implant appears to be safe, well-tolerated, and effective when a new implant is placed for a second year of therapy. Long-term prospective follow-up of treated patients will be essential in order to confirm these results.

\section{References}

[1] P. A. Lee, "Normal ages of pubertal events among American males and females," Journal of Adolescent Health Care, vol. 1, no. 1 , pp. 26-29, 1980.
[2] P. A. Boepple, M. J. Mansfield, M. E. Wierman, et al., "Use of a potent, long acting agonist of gonadotropin-releasing hormone in the treatment of precocious puberty," Endocrine Reviews, vol. 7, no. 1, pp. 24-33, 1986.

[3] A. M. Pasquino, I. Pucarelli, F. Accardo, V. Demiraj, M. Segni, and R. Di Nardo, "Long-term observation of 87 girls with idiopathic central precocious puberty treated with gonadotropinreleasing hormone analogs: impact on adult height, body mass index, bone mineral content, and reproductive function," The Journal of Clinical Endocrinology \& Metabolism, vol. 93, no. 1, pp. 190-195, 2008.

[4] E. K. Neely, R. L. Hintz, B. Parker, et al., "Two-year results of treatment with depot leuprolide acetate for central precocious puberty," The Journal of Pediatrics, vol. 121, no. 4, pp. 634-640, 1992.

[5] K. L. Parker, R. G. Baens-Bailon, and P. A. Lee, "Depot leuprolide acetate dosage for sexual precocity," The Journal of Clinical Endocrinology \& Metabolism, vol. 73, no. 1, pp. 50-52, 1991.

[6] H. J. Hirsch, D. Gillis, D. Strich, et al., "The histrelin implant: a novel treatment for central precocious puberty," Pediatrics, vol. 116, no. 6, pp. e798-e802, 2005.

[7] E. A. Eugster, W. Clarke, G. B. Kletter, et al., "Efficacy and safety of histrelin subdermal implant in children with central precocious puberty: a multicenter trial," The Journal of Clinical Endocrinology \& Metabolism, vol. 92, no. 5, pp. 1697-1704, 2007.

[8] W. W. Greulich and S. I. Pyle, Radiographic Atlas of Skeletal Development of the Hand and Wrist, Stanford University Press, Stanford, Calif, USA, 2nd edition, 1959.

[9] A. Badaru, D. M. Wilson, L. K. Bachrach, et al., "Sequential comparisons of one-month and three-month depot leuprolide regimens in central precocious puberty," The Journal of Clinical Endocrinology \& Metabolism, vol. 91, no. 5, pp. 18621867, 2006.

[10] G. J. Kunz, T. I. Sherman, and K. O. Klein, "Luteinizing hormone (LH) and estradiol suppression and growth in girls with central precocious puberty: is more suppression better? Are pre-injection LH Levels useful in monitoring treatment?" The Journal of Pediatric Endocrinology \& Metabolism, vol. 20, no. 11, pp. 1189-1198, 2007. 